\title{
Dominator coloring on anti fuzzy graph
}

\author{
R. Muthuraj ${ }^{1}$ and A. Sasireka ${ }^{2 *}$
}

\begin{abstract}
In this paper, the concept of dominator coloring on anti fuzzy graph is introduced and the fuzzy dominator chromatic number of anti fuzzy graph is characterized. This concept is applied on anti cartesian product of anti fuzzy graphs and obtained the results on them.

Keywords

Anti fuzzy graph, Domination number, Dominator coloring.

AMS Subject Classification 05C72, 05C15, 05C69, 05C76, 05A18.

${ }^{1}$ Assistant Professor, PG \& Research Department of Mathematics, H.H. The Rajah's College, Pudukkottai - 622001, Tamil Nadu, India.

${ }^{2}$ Assistant Professor, Department of Mathematics, PSNA College of Engineering and Technology, Dindigul-624 622, Tamil Nadu, India.

*Corresponding author: ${ }^{1}$ rmr1973@yahoo.co.in; ${ }^{2}$ sasireka.psna@gmail.com

Article History: Received 22 November 2018; Accepted 09 May 2019

(C)2019 MJM.
\end{abstract}

\section{Contents}

Introduction ................................ 217

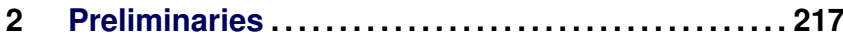

3 Dominator Coloring on Strong Anti Fuzzy Graph 218

4 Dominator coloring on Anti Fuzzy Graph with weak edges...................................221

5 Dominator Coloring on Anti Cartesian Product of Anti Fuzzy Graphs................................221

Conclusion ................................ 222

References .............................. 222

\section{Introduction}

Dominator coloring plays a vital role in biomedical applications such as predicting the cancer cells via buckyball and telecommunication networks, distributed computing via circulant graph. In 2005, Munoz et.al., delivered the idea of coloring on fuzzy graph[9] and described the chromatic number of fuzzy graph. In 2006, Ralucca Michelle Gera established the fuzzy dominator coloring[6]. In the same time, Eslachi and Onagh pioneered the concept of fuzzy chromatic number as the number of partition of the color classes[3]. In 2015, R.Jahir Hussian and K.S.Kanzuli Fathima conferred fuzzy dominator coloring of fuzzy graph[7]. In 2012, Muhammad Akram introduced the concept of an anti fuzzy structures on graphs and characterized some basic concept of con- nected anti fuzzy graph[8]. In 2016, R.Seethalakshmi and R.B.Gnanajothi introduced the definition of anti podal on anti fuzzy graph[20]. In 2017, this concept further developed by R.Muthuraj and A. Sasireka[10-12]. They introduced some types of anti fuzzy graphs and applied some operations on them and also derived some domination parameter on anti fuzzy graph[14, 15]. In 2018, Rifayathali et.al.,[17, 19] introduced the concept of coloring on anti fuzzy graph. In this paper, anti fuzzy graphs are considered without loops and multiple edges. $\mathrm{V}$ is a finite and non empty set. $\mathrm{m}$ and $\mathrm{n}$ are the number of vertices and edges of an anti fuzzy graph. The dominator coloring is defined on anti fuzzy graph. These concepts are applied on anti cartesian product of anti fuzzy graphs.

\section{Preliminaries}

In this section, basic concepts of anti fuzzy graph are discussed. Notations and more formal definitions are followed as in $[3,8,10-12,14,16,20]$.

Definition 2.1. [20] An anti fuzzy graph $G_{A}=(\sigma, \mu)$ is a pair of functions $\sigma: V \rightarrow[0,1]$ and $\mu: V \times V \rightarrow[0,1]$, with $\mu(u, v) \geq \sigma(u) \vee \sigma(v)$ for all $u, v \in V$.

Note. $\mu$ is considered as reflexive and symmetric. In all examples $\sigma$ is chosen suitably. i.e., undirected anti fuzzy graphs are only considered. Without loss of generality, let us simply use the letter $\mathrm{G}_{A}$ to denote an anti fuzzy graph. 
Definition 2.2. [8] Let $G_{A}=(V, \sigma, \mu)$ be an anti fuzzy graph. The order $p$ of $G_{A}$ is defined as $p=\sum_{x \in V} \sigma(x)$. The size $q$ is, $q$ $=\sum_{x y \in E} \mu(x, y)$. It is denoted by $O(G)$ and $S(G)$.

Definition 2.3. [12] Two vertices $u$ and $v$ in $G_{A}$ are called adjacent if (1/2) $[\sigma(u) \vee \sigma(v)] \leq \mu(u, v)$.

Definition 2.4. [10] The anti complement of anti fuzzy graph $G_{A}=(\sigma, \mu)$ is an anti fuzzy graph $\overline{G_{A}}=(\bar{\sigma}, \bar{\mu})$ where $\bar{\sigma}=$ $\sigma$ and $\bar{\mu}(u, v)=\mu(u, v)-(\sigma(u) \vee \sigma(v))$ for all $u, v$ in $V$.

Definition 2.5. [20] An anti fuzzy graph $G_{A}=(\sigma, \mu)$ is a strong anti fuzzy graph of $\mu(u, v)=\sigma(u) \vee \sigma(v)$ for all $(u, v) \in \mu^{*}$ and $G_{A}$ is a complete anti fuzzy graph if $\mu(u, v)=\sigma(u) \vee \sigma(v)$ for all $(u, v) \in \mu^{*}$ and $u, v \in \sigma^{*}$. Two vertices $u$ and $v$ are said to be neighbors if $\mu(u, v)>0$.

Definition 2.6. [12] An edge $e=\{u, v\}$ of an anti fuzzy graph $G_{A}$ is called an effective edge if $\mu(u, v)=\sigma(u) \vee \sigma(v)$.

Definition 2.7. [11] $u$ is a vertex in an anti fuzzy graph $G_{A}$ then $N(u)=\{v:(u, v)$ is an effective edge $\}$ is called the open neighborhood of $u$ and $N[u]=N(u) \cup\{u\}$ is called closed neighborhood of $u$.

Definition 2.8. $[8,11] A$ path $P_{A}$ in an anti fuzzy graph is a sequence of distinct vertices $u_{0}, u_{1}, u_{2}, \ldots, u_{n}$ such that $\mu\left(u_{i-1}, u_{i}\right)>0,1 \leq i \leq n$. Here $n \geq 0$ is called the length of the path $P_{A}$. The consecutive pairs $\left(u_{i-1}, u_{i}\right)$ are called the edges of the path.

Definition 2.9. [8] A cycle in $G_{A}$ is said to be an anti fuzzy cycle if it contains more than one weakest vertex. It is denoted by $C_{A}$.

Definition 2.10. [11] Let $G_{A}^{*}=G_{A_{1}}^{*} \times G_{A_{2}}^{*}=\left(V, E^{\prime}\right)$ be the anti cartesian product of anti fuzzy graphs where $V=V_{1} \times$ $V_{2}$ and $E^{\prime}=\left\{\left(\left(u_{1}, u_{2}\right),\left(u_{1}, v_{2}\right)\right) / u_{1} \in V_{1},\left(u_{2}, v_{2}\right) \in E_{2}\right\} \cup$ $\left.\left(\left(u_{1}, w_{2}\right),\left(v_{1}, w_{2}\right)\right) / w_{2} \in V_{2},\left(u_{1}, v_{1}\right) \in E_{1}\right\}$. Then the anti cartesian product of two anti fuzzy graphs, $G_{A}=G_{A_{1}} \times G_{A_{2}}$ : $\left(\sigma_{1} \times \sigma_{2}, \mu_{1} \times \mu_{2}\right)$ is an anti fuzzy graph and is defined by

$\left(\sigma_{1} \times \sigma_{2}\right)\left(u_{1}, u_{2}\right)=\max \left\{\sigma_{1}\left(u_{1}\right), \sigma_{2}\left(u_{2}\right)\right\}$ for all $\left(u_{1}\right.$, $\left.u_{2}\right) \in V$,

$\left(\mu_{1} \times \mu_{2}\right)\left(\left(u_{1}, u_{2}\right),\left(u_{1}, v_{2}\right)\right)=\max \left\{\sigma_{1}\left(u_{1}\right), \mu_{2}\left(u_{2}, v_{2}\right)\right\}$ for all $u_{1} \in V_{1}$ and $\left(u_{2}, v_{2}\right) \in E_{2}$,

$\left(\mu_{1} \times \mu_{2}\right)\left(\left(u_{1}, w_{2}\right),\left(v_{1}, w_{2}\right)\right)=\max \left\{\sigma_{2}\left(w_{2}\right), \mu_{1}\left(u_{1}, v_{1}\right)\right\}$ for all $w_{2} \in V_{2}$ and $\left(u_{1}, v_{1}\right) \in E_{1}$.

Then the anti fuzzy graph $G_{A}=\left(\sigma_{1} \times \sigma_{2}, \mu_{1} \times \mu_{2}\right)$ is said to be the anti cartesian product of anti fuzzy graphs $G_{A_{1}}=\left(\sigma_{1}\right.$, $\left.\mu_{1}\right)$ and $G_{A_{2}}=\left(\sigma_{2}, \mu_{2}\right)$.

Definition 2.11. [12] Every vertex in an anti fuzzy graph $G_{A}$ has unique fuzzy values then $G_{A}$ is said to be v-nodal anti fuzzy graph. i.e. $\sigma(u)=c$ for all $u \in V\left(G_{A}\right)$.

Definition 2.12. [12] Every edge in an anti fuzzy graph $G_{A}$ has unique fuzzy values then $G_{A}$ is said to be e-nodal anti fuzzy graph. i.e. $\mu(u, v)=c$ for all $(u, v) \in E\left(G_{A}\right)$.
Definition 2.13. [12] Every vertices and edges in an anti fuzzy graph $G_{A}$ have the unique fuzzy values then $G_{A}$ is called as uninodal anti fuzzy graph. If $\sigma(u)=c_{1}$ and $\mu(u, v)=c_{2}$ in an anti fuzzy graph $G_{A}$ then $G_{A}$ is called as binodal anti fuzzy graph.

Definition 2.14. $[11,14]$ The strong neighbourhood of an edge $e_{i}$ in an anti fuzzy graph $G_{A}$ is $N_{s}\left(e_{i}\right)=\left\{e_{j} \in E(G) / e_{j}\right.$ is an effective edge with $\vee N\left(e_{i}\right)$ in $G_{A}$ and adjacent to $\left.e_{i}\right\}$

Definition 2.15. [14] An edge $e=\{u, v\}$ of an anti fuzzy graph $G_{A}$ is called an weak edge if $\mu(u, v) \neq \sigma(u) \vee \sigma(v)$.

Definition 2.16. [16] $G_{A}$ is an anti fuzzy graph and $u, v \in$ $V\left(G_{A}\right)$. If $v$ is said to be a support vertex in $G_{A}$ then $v$ is adjacent to at least one end vertex $u$.

Definition 2.17. [17] Let $G_{A}$ be an anti fuzzy graph. A family $c=\left\{c_{1}, c_{2}, \ldots, c_{k}\right\}$ of fuzzy sets as a set $V$ is called a $k$-vertex coloring of $G_{A}=(V, \sigma, \mu)$ if

1. $\vee c_{i}(x)=\sigma(x)$

2. $c_{i} \wedge c_{j}=0$

3. For every strong edge xy of $G_{A}, \min \left\{c_{i} \sigma((x)), c_{i} \sigma((y))\right.$ \}$=0,(1 \leq i \leq k)$. The least value of $k$ for which $G$ has $k$-vertex coloring denoted by $\chi\left(G_{A}\right)$, is called the chromatic number of the anti fuzzy graph $G_{A}$.

\section{Dominator Coloring on Strong Anti Fuzzy Graph}

In this section, dominator coloring is applied on strong anti fuzzy graph and the fuzzy dominator chromatic number on connected anti fuzzy graph which has strong edges only is determined. The membership value of the color class is 1 . So, assigning a color to the vertices of an anti fuzzy graph as $1,2,3, .$. instead of $\operatorname{Red}(\mathrm{R}), \operatorname{Green}(\mathrm{G})$, Blue(B), Yellow(Y), etc. That is, every number $(1,2,3, .$.$) represent a one color class of$ $\mathrm{G}_{A}$ and the fuzzy value of color class is assigned the value 1 . Depending upon the nature of dominator coloring, a vertex is classified as $\chi_{f d}$-good and C-bad vertex. By using this characterization the anti fuzzy graph is also classified as $\chi_{f d^{-}}$ good and $\chi_{f d}$-excellent. The bounds are obtained for them and derived some theorems of dominator chromatic number on anti fuzzy graph.

Definition 3.1. A colored anti fuzzy graph $G_{A}$ is an anti fuzzy graph in which each vertex of $G_{A}$ is assigned a color from color class $c$. A properly colored anti fuzzy graph is a colored anti fuzzy graph whose color assignments conform to the coloring rules applied to the anti fuzzy graph. Fuzzy chromatic number of an anti fuzzy graph $G_{A}$ is the least number of distinct colors and is denoted by $\chi\left(G_{A}\right)$.

Definition 3.2. A fuzzy dominator coloring of an anti fuzzy graph $G_{A}$ is a proper fuzzy coloring in which each vertex of $G_{A}$ dominates every vertex of atleast some color class. 
Fuzzy dominator chromatic number of an anti fuzzy graph is the minimum number of color classes in a dominator fuzzy coloring of $G_{A}$. It is denoted by $\chi_{f d}\left(G_{A}\right)$.

Definition 3.3. $C$ is the partition of independent sets of vertices of an anti fuzzy graph $G_{A}$ in which $u \in D$ and $v \in V \backslash D$ is in distinct partition of color class $C$ and each vertex of $G_{A}$ dominates every vertex of atleast one color class. Such $C$ is called the anti fuzzy dominator partition of $G_{A}$.

\section{Example 3.4.}

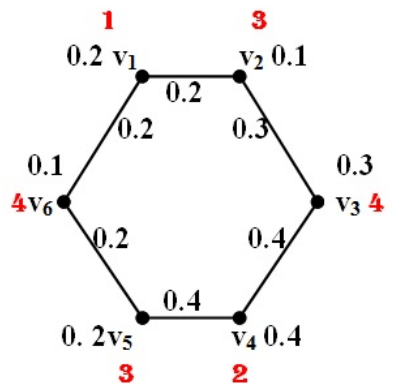

(a)

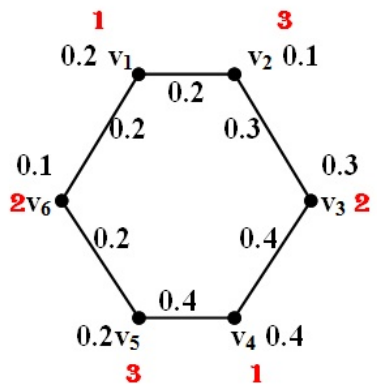

(b)
Figure 1. (a)Anti Fuzzy Graph $\mathrm{G}_{A}$ with dominator coloring; (b) Anti fuzzy graph $\mathrm{G}_{A}$ with coloring

In Figure 1(a), $G_{A}$ is an anti fuzzy graph with proper coloring. The color classes of Figure 1(a) are $c_{1}, c_{2}, c_{3}$ and $c_{4}$. Where $c_{1}=\left\{v_{1}\right\}, c_{2}=\left\{v_{4}\right\}, c_{3}=\left\{v_{2}, v_{5}\right\}$ and $c_{4}=\left\{v_{3}\right.$, $\left.v_{6}\right\}$. The vertices $v_{1}$ and $v_{4}$ dominates the remaining vertices and the vertices in the color class of $c_{3}$ and $c_{4}$ dominate all the vertices of atleast any one of color classes $c_{1}$ and $c_{2}$. Therefore, Figure 1(a) - $G_{A}$ is an anti fuzzy graph with proper dominator coloring.

In Figure 1(b), $G_{A}$ is an anti fuzzy graph with proper coloring and the color classes are $c_{1}, c_{2}$ and $c_{3}$. Where $c_{1}=\left\{v_{1}, v_{4}\right\}, c_{2}=\left\{v_{3}, v_{6}\right\}$ and $c_{3}=\left\{v_{2}, v_{5}\right\}$. No vertex of any color classes dominate atleast one color class of $G_{A}$. Therefore, Figure 1(b)- $G_{A}$ is not a proper dominator coloring.

Definition 3.5. A vertex $v \in V\left(G_{A}\right)$ is called $\chi_{f d-g o o d}$ if a vertex $v$ belongs to some $C$-partition( $\chi$-partition) otherwise $v$ is said to be $C$-bad vertex.

An anti fuzzy graph $G_{A}$ is called $\chi_{f d}$-excellent anti fuzzy graph if every vertex of $G_{A}$ is $\chi_{f d}$-good.

Example 3.6. Now, consider the following Figure 2, to determine the fuzzy dominator chromatic number of an anti fuzzy graph.

In Figure 2, the dominating set of $\mathrm{G}_{A}$ is $\mathrm{D}=\left\{\mathrm{v}_{1}, \mathrm{v}_{7}\right\}$.

The color class is $c=\left\{c_{1}, c_{2}, c_{3}, c_{4}\right\}=\left\{\left\{v_{1}\right\}^{1},\left\{v_{7}\right\}^{1},\left\{v_{2}\right.\right.$, $\left.\left.\mathrm{v}_{4}, \mathrm{v}_{5}\right\}^{1},\left\{\mathrm{v}_{3}, \mathrm{v}_{6}, \mathrm{v}_{8}\right\}^{1}\right\}$

Therefore, $\chi_{f d}\left(\mathrm{G}_{A}\right)=1+1+1+1=4$.

$$
\begin{aligned}
& \mathrm{c}_{1}\left(\mathrm{v}_{i}\right)=\left\{\begin{array}{l}
0.8, \text { if } i=1 \\
0, \text { otherwise }
\end{array}\right. \\
& \mathrm{c}_{2}\left(\mathrm{v}_{i}\right)=\left\{\begin{array}{l}
0.7, \text { if } i=7 \\
0, \text { otherwise }
\end{array}\right.
\end{aligned}
$$

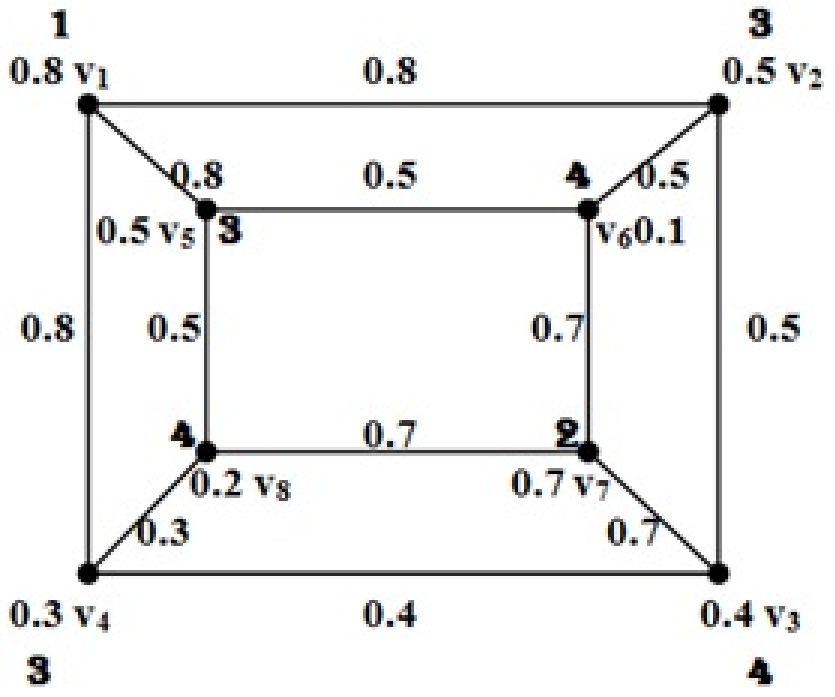

Figure 2. An Anti Fuzzy Graph $\mathrm{G}_{A}$

$$
\begin{gathered}
\mathrm{c}_{3}\left(\mathrm{v}_{i}\right)=\left\{\begin{array}{l}
0.5, \text { if } i=2,5 \\
0.3, \text { if } i=4 \\
0, \text { otherwise }
\end{array}\right. \\
\quad \mathrm{c}_{4}\left(\mathrm{v}_{i}\right)=\left\{\begin{array}{l}
0.1, \text { if } i=6 \\
0.2, \text { if } i=3
\end{array}\right. \\
0, \text { otherwise }
\end{gathered}
$$

Here the vertices $v_{1}, v_{2}, \ldots, v_{8}$ dominate atleast one of the color classes are $c_{1}, c_{2}$ of $G_{A}$. Therefore, $\chi_{f d}\left(G_{A}\right)=4$.

The anti fuzzy dominator partition of Figure 2 is, $\mathrm{C}=\left\{\mathrm{C}_{1}, \mathrm{C}_{2}, \mathrm{C}_{3}, \mathrm{C}_{4}\right\}$.

Where $C_{1}=\left(\left\{\mathrm{v}_{1}\right\},\left\{\mathrm{v}_{7}\right\},\left\{\mathrm{v}_{2}, \mathrm{v}_{4}, \mathrm{v}_{5}\right\},\left\{\mathrm{v}_{3}, \mathrm{v}_{6}, \mathrm{v}_{8}\right\}\right)$

$\mathrm{C}_{2}=\left(\left\{\mathrm{v}_{2}\right\},\left\{\mathrm{v}_{8}\right\},\left\{\mathrm{v}_{4}, \mathrm{v}_{5}, \mathrm{v}_{7}\right\},\left\{\mathrm{v}_{1}, \mathrm{v}_{3}, \mathrm{v}_{6}\right\}\right)$

$\mathrm{C}_{3}=\left(\left\{\mathrm{v}_{3}\right\},\left\{\mathrm{v}_{5}\right\},\left\{\mathrm{v}_{2}, \mathrm{v}_{4}, \mathrm{v}_{7}\right\},\left\{\mathrm{v}_{1}, \mathrm{v}_{6}, \mathrm{v}_{8}\right\}\right)$

$\mathrm{C}_{4}=\left(\left\{\mathrm{v}_{4}\right\},\left\{\mathrm{v}_{6}\right\},\left\{\mathrm{v}_{2}, \mathrm{v}_{7}, \mathrm{v}_{5}\right\},\left\{\mathrm{v}_{3}, \mathrm{v}_{1}, \mathrm{v}_{8}\right\}\right)$

Every vertex of $\mathrm{G}_{A}$ appears in a singleton in $\chi$-partition. Hence $\mathrm{G}_{A}$ is $\chi$-excellent.

Every vertex of $\mathrm{G}_{A}$ dominates atleast one of the color classes of $\mathrm{c}_{i}$ and also appears in singleton of $\chi$-partition. Hence $\mathrm{G}_{A}$ is $\chi_{f d}$-excellent.

\section{Proposition 3.7 .}

1. If an anti fuzzy graph $G_{A}$ has no edges with $m$ vertices then fuzzy dominator chromatic number is $m$.

2. For any bipartite anti fuzzy graph with atleast one edge, the fuzzy dominator chromatic number is at least $|D|+1$. Where $|D|$ is the number of vertices in a minimal dominating set of an anti fuzzy graph $G_{A}$.

Example 3.8. Consider the following Figure 3, for characterizing chromatic number of an anti fuzzy graph. 


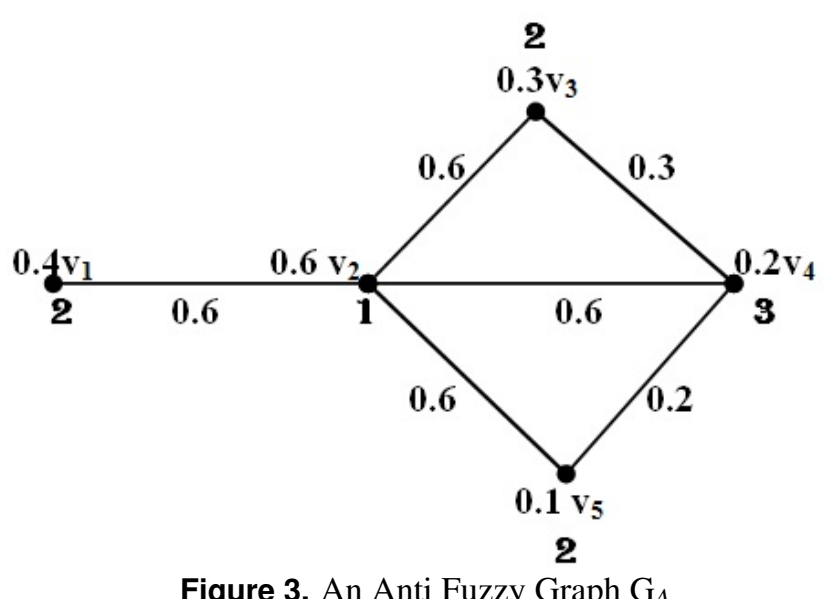

In Figure 3, $C_{1}=\left(\left\{v_{2}\right\},\left\{v_{1}, v_{3}, v_{5}\right\},\left\{v_{4}\right\}\right)$. The vertices $v_{1}, v_{3}, v_{5}$ do not appears in singleton of $\chi$-partition. Hence $G_{A}$ is not $\chi_{f d}$-excellent.

Theorem 3.9. For any connected anti fuzzy graph, $\chi_{f d}\left(G_{A}\right)$ $\geq|D|+1$.

Proof. Suppose $\mathrm{N}(\mathrm{V}(\mathrm{D}))=1$ and any one of the color in color class ' $\mathrm{c}_{i}$ ' is assigned except the color assigned by the dominating vertices in $\mathrm{D}$. All the vertices in $\mathrm{D}$ is assigned with individual color from color class $\mathrm{c}_{i}$. Therefore, the color class should contain $|D|$ colors. Let $\mathrm{v}_{n} \in \mathrm{V} \backslash \mathrm{D}$ where $\mathrm{v}_{n}$ is adjacent with atleast one vertex in $\mathrm{D}$. So, the vertex $\mathrm{v}_{n}$ is assigned with $\mathrm{c}_{|D|+1}^{\text {th }}$ color and this vertex dominate any one of color class of $\mathrm{G}_{A}$.

Therefore, $\chi_{f d}\left(\mathrm{G}_{A}\right)=|D|+1 .---\rightarrow(1)$

If $\mathrm{N}(\mathrm{V}(\mathrm{D})) \geq 1$ then all the vertices in $\mathrm{D}$ is assigned with an individual color from color class $\mathrm{c}_{i}$. Therefore, the color class should contain $|D|$ colors. Assume that the vertices $\mathrm{v}_{n}, \mathrm{v}_{n+1} \in \mathrm{V} \backslash \mathrm{D}$. To construct a proper dominator coloring, these vertices are colored with $\mathrm{c}_{|D|+1}$ th and $\mathrm{c}_{|D+2|}$ th color. Since $\mathrm{v}_{n}$ adjacent to $\mathrm{v}_{n+1}$.

Therefore, $\chi_{f d}\left(\mathrm{G}_{A}\right)=|D|+2 .---\rightarrow(2)$

Similarly repeat this process until all the vertices in $\mathrm{V} \backslash \mathrm{D}$ is assigned with some colors.

Hence,from (1) and (2), $\chi_{f d}\left(\mathrm{G}_{A}\right) \geq|D|+1$.

Theorem 3.10. If $G_{A}$ is an anti fuzzy path with $m \geq 5$ vertices then $\chi_{f d}\left(G_{A}\right)=|D|+2$.

Proof. Let $\mathrm{v}_{i}$ be the internal vertex in an anti fuzzy path $\mathrm{G}_{A}$. It dominates $\mathrm{v}_{i-1}, \mathrm{v}_{i+1}$. Therefore, $\mathrm{v}_{i}$ may be assigned with color $\mathrm{c}_{1}$ in $\mathrm{c}$. The vertices $\mathrm{v}_{i-1}, \mathrm{v}_{i+1}$ may be assigned with color $\mathrm{c}_{|D|+1}$ th in c. If $\mathrm{v}_{i+2} \notin \mathrm{D}$ and $\mathrm{v}_{i+1}$ adjacent with $\mathrm{v}_{i+2}$ then $\mathrm{v}_{i+2}$ is assigned with color $\mathrm{c}_{|D|+2}$.

Suppose $\mathrm{v}_{i+2} \in \mathrm{D}$ then $\mathrm{v}_{i+2}$ is assigned with color $\mathrm{c}_{2} \leq$ $\mathrm{c}_{|D|}$. Therefore, the vertices in $\mathrm{D}$ are assigned with individual color and dominate any color classes of $\mathrm{c}$. The vertices in $\mathrm{V} \backslash \mathrm{D}$ is assigned with alternate color of $\mathrm{c}_{|D|+1}, \mathrm{c}_{|D|+2}$. Since any pair of vertices in $\mathrm{V} \backslash \mathrm{D}$ which are adjacent to each other.

Hence $\chi_{f d}\left(\mathrm{G}_{A}\right)=|D|+2$.

\section{Proposition 3.11.}

1. If $G_{A}$ is an anti fuzzy path with $m<5$ vertices then $\chi_{f d}\left(G_{A}\right)=|D|+\left\lceil\frac{m}{3}\right\rceil$.

2. For a uninodal anti fuzzy graph $G_{A}, \chi_{f d}\left(G_{A}\right)=\left\lceil\frac{2 m}{3}\right\rceil$.

3. If $G_{A}$ is e-nodal anti fuzzy graph with effective edges only then $\chi_{f d}\left(G_{A}\right) \geq|D|+1$.

Remark 3.12. Consider that the $|D|$ is the number of vertices in a minimal dominating set of an anti fuzzy graph $G_{A}$.

1. If an anti fuzzy path contains two vertices then $|D|=1$, $\left\lceil\frac{2}{3}\right\rceil=1$. Therefore $\chi_{f d}\left(G_{A}\right)=1+1=2$.

2. If an anti fuzzy path contains 3 vertices (say $u_{1}, u_{2}$, $\left.u_{3}\right)$ then $|D|=1$ and $|V \backslash D|=2$. That is, the internal vertex $u_{2} \in D$ and assigned with color $c_{1}$. The vertices in $V \backslash D$ are non adjacent to each other and a strong neighbor to a vertex $u_{2}$ in $D$. So the vertices $u_{1}$ and $u_{3}$ are assigned with same color $c_{2} .|D|=1,\left\lceil\frac{3}{3}\right\rceil=1$. Therefore, $\chi_{f d}\left(G_{A}\right)=1+1=2$.

Theorem 3.13. If $G_{A}$ is a anti fuzzy tree with $m \geq 3$ vertices then $\chi_{f d}\left(G_{A}\right)=|S|+1$, where $S$ is the set of all support vertices in $G_{A}$.

Proof. Consider that $\mathrm{G}_{A}$ is an anti fuzzy tree with atleast 3 vertices. Then $\mathrm{G}_{A}$ has atleast one support vertex (say $\mathrm{u}$ ). The vertex u is colored with color 1 from $|S|$ color classes which also dominate other vertices in $\mathrm{G}_{A}$. The vertices which are adjacent to $u$ but not a leaf of $\mathrm{G}_{A}$ is assigned with color $(|S|-1)^{t h}$. Similarly, repeat this procedure until all the support vertices are assigned with some color from $|S|$ color classes. Finally, all the pendent vertices are assigned with $(|S|+1)^{t h}$ color. Hence $\chi_{f d}\left(\mathrm{G}_{A}\right)=|S|+1$.

Theorem 3.14. For any connected irregular anti fuzzy graph $G_{A}$,

1. $\chi\left(G_{A}\right) \geq \Delta I_{c}$.

2. $\chi_{f d}\left(G_{A}\right) \geq \Delta I_{c}+1$.

where $I_{C}$ is the set of all incident vertices of a vertex $v$ in $G_{A}$ and $\Delta I_{c}$ is the maximum number of incident vertices of a vertex in $G_{A}$.

Proof. Consider that $\mathrm{G}_{A}$ is a connected irregular anti fuzzy graph. That is, any one of the vertices in $\mathrm{G}_{A}$ has number of incident vertices as $\Delta I_{c}$. For coloring an anti fuzzy graph $\mathrm{G}_{A}$, a vertex is arbitrarily chosen (say u) which have $\Delta I_{c}$ neighbors and assign with color 1 . All the vertices in $\mathrm{G}_{A}$, except $\mathrm{u}$ have atmost $\Delta I_{c}$ neighbors. Consider a vertex $\mathrm{v} \in \mathrm{V}\left(\mathrm{G}_{A}\right) \backslash\{\mathrm{u}\}$ which have $\Delta I_{c}$ neighbors. This vertex is colored with $\left(\Delta I_{c^{-}}\right.$ $1)^{\text {th }}$ color. Since $\mathrm{u}$ is adjacent with $\mathrm{v}$. Repeat this process until all the vertices are assigned with a color. Finally, the anti fuzzy graph $\mathrm{G}_{A}$ is colored using $\Delta I_{c}$ colors. Hence $\chi\left(\mathrm{G}_{A}\right)$ $\geq \Delta I_{c}$. 
Similarly, choose another vertex $\mathrm{w} \in \mathrm{V}\left(\mathrm{G}_{A}\right) \backslash\{\mathrm{u}, \mathrm{v}\}$ which have $\Delta I_{c}$ neighbors and colored the vertex $\mathrm{w}$ by $\left(\Delta I_{c}-1\right)^{t h}$ color. Since $\mathrm{w}$ is adjacent with u but not with $\mathrm{v}$. Repeat this procedure until all vertices(having $\Delta I_{c}$ neighbors) are colored. Finally, get some vertices which does not have the $\Delta I_{c}$ neighbors. Such vertex can colored by $\left(\Delta I_{c}+1\right)^{t h}$ color. Hence the vertices which are having $\Delta I_{c}$ neighbors should be a member in dominating set of $\mathrm{G}_{A}$. The color class which contains these vertices should form a dominator coloring of $\mathrm{G}_{A}$. Therefore, $\chi_{f d}\left(\mathrm{G}_{A}\right) \geq \Delta I_{c}+1$.

Theorem 3.15. For any connected $k$-regular anti fuzzy graph $G_{A}, \quad \chi_{f d}\left(G_{A}\right)>\Delta I_{c}+1$.

Proposition 3.16.

1. For any connected anti fuzzy graph $G_{A}, \chi\left(G_{A}\right) \leq \chi_{f d}\left(G_{A}\right)$

2. Complete anti fuzzy graph is $\chi_{f d}$-excellent.

3. Anti complement of complete anti fuzzy graph is also $\chi_{f d}$-excellent.

4. Cyclic anti fuzzy graph $G_{A}$ is $\chi_{f d}$-excellent.

5. If anti fuzzy graph $G_{A}$ is star then $G_{A}$ is $\chi_{f d}$-excellent.

6. Any connected k-regular anti fuzzy graph $G_{A}$ is $\chi_{f d^{-}}$ excellent.

\section{Dominator coloring on Anti Fuzzy Graph with weak edges}

In this section, the dominator chromatic number is derived for an anti fuzzy graph which has atleast one weakest edges. The color class which contains only the strong edges is assigned with membership fuzzy value as 1 . If the color class has atleast one weakest edges then the membership value of color class is defined as follows.

Definition 4.1. Let $u, v$ be twp vertices in $G_{A}$ and $u, v$ are adjacent with one weakest edge(e) then the fuzzy color values of $u$ is defined as $1-[\mu(u, v)-\vee(\sigma(u), \sigma(v))]$ and the color class value is minimum of fuzzy color values of color class $c_{i}$.

Example 4.2. In Figure 4, $G_{A}$ is an anti fuzzy graph with one weak edge $\left(v_{1}, v_{5}\right)$.

The vertices $v_{5}$ and $v_{6}$ dominate all other vertices in $G_{A}$ and maximum fuzzy cardinality of a dominating set with minimum number of vertices is obtained with these vertices. Therefore, it forms a minimal dominating set. So, these vertices are assigned with individual colors such as 1 and 2. The corresponding color class is $c_{1}, c_{2}$ and the membership value is 1 .

Next, colors are assigned to the remaining vertices with color class $3,4, \ldots$ Randomly choose a vertex $v_{1}$, which is adjacent with $v_{5}$ but the edge $\left(v_{1}, v_{5}\right)$ is weak edge in $G_{A}$.

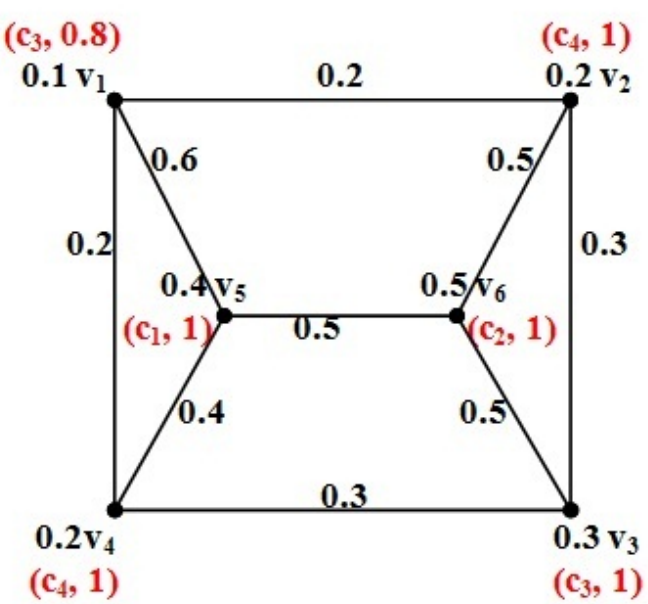

Figure 4. An Anti Fuzzy Graph $\mathrm{G}_{A}$

So the vertex $v_{1}$ is assigned with color 3 whose fuzzy color membership value is $1-[0.6-(0.1 \vee 0.4)]=1-(0.6-0.4)=$ $1-(0.6-0.4)=1-0.2=0.8$.

Then the vertices $v_{2}$ and $v_{4}$ are adjacent with $v_{1}$ as strong neighbors. Such vertices are assigned with color 4 . The vertex $v_{3}$ is adjacent with $v_{2}$ and $v_{4}$ as a strong neighbor. Color 3 is assigned with fuzzy color membership value 1. $c=\left\{c_{1}, c_{2}, c_{3}\right.$, $\left.c_{4}\right\}=\left\{\left\{v_{5}\right\}^{1},\left\{v_{6}\right\}^{1},\left\{v_{1}, v_{3}\right\}^{0.8},\left\{v_{2}, v_{4}\right\}^{1}\right\}$

Therefore, $\chi_{f d}\left(G_{A}\right)=|c|=1+1+0.8+1=3.8$

\section{Proposition 4.3.}

1. If $G_{A}$ is e-nodal anti fuzzy graph with weak edges then $\chi_{f d}\left(G_{A}\right) \geq|D|+\frac{m}{2}$.

2. In e-nodal(v-nodal) anti fuzzy graph with weak edges, the vertex incident with weak edges is assigned the fuzzy color value is greater than 1.

\section{Dominator Coloring on Anti Cartesian Product of Anti Fuzzy Graphs}

In this section, the dominator coloring is applied on anti cartesian product of anti fuzzy graphs and obtained the bounds of fuzzy dominator chromatic number on anti cartesian product of anti fuzzy graphs. Anti cartesian product of anti fuzzy graphs are considered as strong anti fuzzy graph and the fuzzy value of the color class is 1 . If $G_{A_{1}} \times G_{A_{2}}$ has weak edges then the fuzzy value is calculated by $1-[\mu(\mathrm{u}, \mathrm{v})-\vee(\sigma(u), \sigma(v))]$. In this section, all $G_{A_{1}}$ and $G_{A_{2}}$ are considered as strong anti fuzzy graph.

Definition 5.1. [11] Let $G_{A}$ be an anti cartesian product of anti fuzzy graphs $G_{A_{1}}$ and $G_{A_{2}}$ where $G_{A_{1}}=\left(\sigma_{1}, \mu_{1}\right)$ and $G_{A_{2}}=$ $\left(\sigma_{2}, \mu_{2}\right)$ then $G_{A}=\left(\sigma_{1} \times \sigma_{2}, \mu_{1} \times \mu_{2}\right)$ is an anti fuzzy graph.

Theorem 5.2. Let $G_{A_{1}}$ and $G_{A_{2}}$ be the anti fuzzy paths on $m_{1}$ and $m_{2}$ vertices. If the anti Cartesian product $G_{A_{1}} \times G_{A_{2}}$ of $G_{A_{1}}$ and $G_{A_{2}}$ is an anti fuzzy graphs on $m_{1} m_{2}$ vertices then 
$\chi_{f d}\left(G_{A_{1}} \times G_{A_{2}}\right) \geq|D|+2$, where $|D|$ is the number of vertices in a minimal dominating set of $G_{A}$.

Theorem 5.3. Let $G_{A}=G_{A_{1}} \times G_{A_{2}}$ be an anti cartesian product of anti fuzzy graphs $G_{A_{1}}$ and $G_{A_{2}}$ then $\chi_{f d}\left(G_{A_{1}} \times G_{A_{2}}\right) \geq$ $\max \left\{\chi_{f d}\left(G_{A_{1}}\right), \chi_{f d}\left(G_{A_{2}}\right)\right\}$.

Proof. By the definition of anti cartesian product of anti fuzzy graphs, any two vertices are adjacent in $G_{A_{1}}$ or in $G_{A_{2}}$ if and only if the corresponding pair of vertices are adjacent in $G_{A_{1}} \times G_{A_{2}}$. That is, by the definition of anti cartesian product of anti fuzzy graphs, more adjacency exists in $G_{A_{1}} \times G_{A_{2}}$. Assigning the color to the vertices depend on the adjacency in $G_{A_{1}} \times G_{A_{2}}$. Therefore, the number of colors required to the vertices of $G_{A_{1}} \times G_{A_{2}} \geq \max \left\{\chi_{f d}\left(G_{A_{1}}\right), \chi_{f d}\left(G_{A_{2}}\right)\right\}$.

Theorem 5.4. Let $G_{A_{1}}$ and $G_{A_{2}}$ be the anti fuzzy cycles on $m_{1}$ and $m_{2}$ vertices. If the anti cartesian product of anti fuzzy cycle $G_{A}=\left(G_{A_{1}} \times G_{A_{2}}\right)$ is an anti fuzzy graph on $m_{1} m_{2}$ vertices then $\chi_{f d}\left(G_{A_{1}} \times G_{A_{2}}\right) \geq|D|+2$, where $|D|$ is the number of vertices in a minimal dominating set of $G_{A}$.

Proof. Similar proof as in [13] for fuzzy graph

Theorem 5.5. If $G_{A_{1}} \times G_{A_{2}}$ is an anti cartesian product of anti fuzzy cycles with $m_{1} m_{2}$ vertices then $\chi\left(G_{A_{1}} \times G_{A_{2}}\right) \leq$ $\chi_{f d}\left(G_{A_{1}} \times G_{A_{2}}\right)$. Where $\left|V\left(G_{A_{1}}\right)\right|=m_{1},\left|V\left(G_{A_{2}}\right)\right|=m_{2}$

Proof. Every vertex in $G_{A_{1}} \times G_{A_{2}}$ are assigned with some colors in color class $\left\{\mathrm{c}_{1}, \mathrm{c}_{2}, . ., \mathrm{c}_{m}\right\}$ where $\mathrm{m}=\mathrm{m}_{1} \times \mathrm{m}_{2}$. By the definition of proper coloring, adjacent pair of vertices are assigned with distinct colors. Therefore, by the definition of anti cartesian product of anti fuzzy cycles the vertices have maximum 4 strong neighbors and minimum 3 neighbors. Hence $\chi\left(G_{A_{1}} \times G_{A_{2}}\right)=3$. - $>$ (1)

In $G_{A_{1}} \times G_{A_{2}}$, there exists a member in a minimal dominating set is $|D| \geq 3$. Neighbors of the vertex in $\mathrm{V}(\mathrm{D})$, each member may be assigned with same color in some color classes (say cth ${ }_{|D|+1}^{t h}$ color) but a vertex $\left(\mathrm{u}_{i}, \mathrm{v}_{j}\right)$ is a strong neighbor to atleast one dominating vertex and a neighbor to a vertex in $\mathrm{V} \backslash \mathrm{D}$. In such case, the vertex $\left(\mathrm{u}_{i}, \mathrm{v}_{j}\right)$ and $\left(\mathrm{u}_{i}, \mathrm{v}_{j+1}\right)$ is assigned with distinct colors.

Hence $\chi_{f d}\left(G_{A_{1}} \times G_{A_{2}}\right) \geq|D|+2 \longrightarrow>(2)$

From (1) and (2), $\chi\left(G_{A_{1}} \times G_{A_{2}}\right) \leq \chi_{f d}\left(G_{A_{1}} \times G_{A_{2}}\right)$.

Theorem 5.6. Let $G_{A_{1}} \times G_{A_{2}}$ be an anti cartesian product of complete anti fuzzy graphs on $m=m_{1} \times m_{2}$ vertices.

1. For $m_{1}=2$ and $m_{2} \geq m_{1}$ then $\chi_{f d}\left(G_{A_{1}} \times G_{A_{2}}\right) \geq\left(m_{1} \vee\right.$ $\left.m_{2}\right)+1$.

2. For $m_{1}, m_{2}>2$, then $\chi_{f d}\left(G_{A_{1}} \times G_{A_{2}}\right) \geq\left(m_{1} \vee m_{2}\right)+2$.

Example 5.7. consider a complete anti fuzzy graph $K_{2}$. Apply the anti cartesian product on anti fuzzy graphs $K_{2}$ and itself. The resulting anti fuzzy graph is shown in Figure 5.

Here in Figure 5, $D=\left\{\left(u_{1}, v_{2}\right),\left(u_{2}, v_{2}\right)\right\},|D|=2$,

$\gamma=.3+.3=0.6$

Let the family of color classes be $c=\left\{c_{1}, c_{2}, c_{3}, c_{4}\right\}$

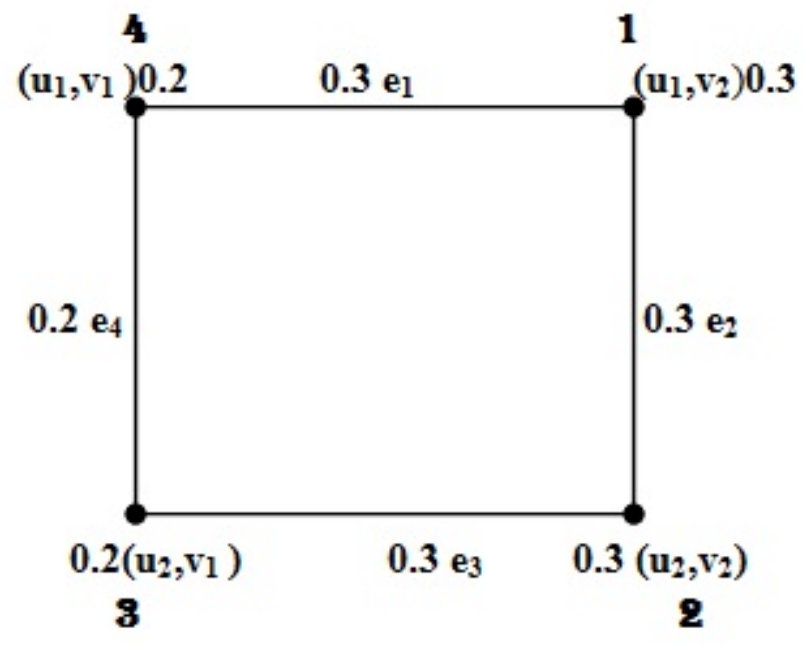

Figure 5. An Anti Fuzzy Graph $\mathrm{G}_{A}=\mathrm{K}_{2} \times \mathrm{K}_{2}$

$$
\begin{aligned}
& c_{1}\left\{\left(u_{i}, v_{j}\right)\right\}=\left\{\begin{array}{r}
0.3, \text { if } i=1, j=2 \\
0, \text { otherwise }
\end{array}\right. \\
& c_{2}\left\{\left(u_{i}, v_{j}\right)\right\}=\left\{\begin{array}{r}
0.3, \text { if } i=2, j=2 \\
0, \text { otherwise }
\end{array}\right. \\
& c_{3}\left\{\left(u_{i}, v_{j}\right)\right\}=\left\{\begin{array}{r}
0.2, \text { if } i=2, j=1 \\
0, \text { otherwise }
\end{array}\right. \\
& c_{4}\left\{\left(u_{i}, v_{j}\right)\right\}=\left\{\begin{array}{r}
0.2, \text { if } i=1, j=1 \\
0, \text { otherwise }
\end{array}\right.
\end{aligned}
$$

Hence all the vertices in $V\left(G_{A_{1}} \times G_{A_{2}}\right)$ belong to some distinct color classes. It satisfies the property of fuzzy dominator

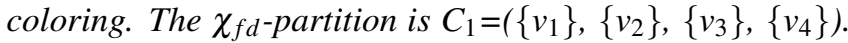

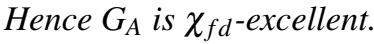

\section{Results}

1. If $G_{A_{1}}$ and $G_{A_{2}}$ are $\chi_{f d}$-excellent then $G_{A_{1}} \times G_{A_{2}}$ need not be $\chi_{f d}$-excellent.

\section{Conclusion}

The dominator coloring is applied on some types of anti fuzzy graph and obtained the bounds of fuzzy dominator chromatic number on anti fuzzy graph. Fuzzy chromatic value is derived for anti fuzzy graph with effective and weak edges. Also, the fuzzy dominator chromatic number is applied on the anti cartesian product of anti fuzzy graph and derived some theorems on them.

\section{References}

[1] S. Arumugam, Jay bagga and K.M.Raja chandrasekar, On dominator colorings in graphs, Proc. Indian Acad. Sci., (Math. Sci.) 122(4)(November 2012), 560-571.

[2] C. Berge, Theorey of graphs, dover Publications, Inc.Mineola, New York, 2001. 
[3] C. Eslachi, B. N. Onagh, Vertex strength of fuzzy graphs, International journal of Mathematics and mathematical sciences (2006).

[4] R. Gera, On Dominator coloring in Graphs, Graph Theory Notes of New York LII, (2007), 25-30.

[5] R. Gera,On dominator colorings in bipartite graphs, (ITNG'07)IEEE,(2007), 1-6.

[6] R. Gera, S. Horton and C. Ramussen, "Dominator colorings and safe clique partitions", Congressus Numerantium, 81(2006), 19-20.

[7] R. jahir Hussian and K.S. Kanzol Fathima, On Fuzzy Dominator coloring in Fuzzy Graphs, Applied Mathematical Sciences, 9(23) (2015), 1131-1137.

[8] Muhammad Akram, Anti Fuzzy Structures on Graphs, Middle East journal of Scientific Research, 11(12) (2012), 1641-1648.

[9] S. Munoz, T. Ortuno, J. Ramirez, J. Yanez, Coloring fuzzy graphs, Omega 33(3) (2005), 211-221.

[10] R. Muthuraj and A. Sasireka, On Anti Fuzzy Graph, Advances in Fuzzy Mathematics, 12(5) (2017), 1123-1135.

[11] R. Muthuraj and A. Sasireka, Some Characterization on Operations of Anti Fuzzy Graphs", International Conference on Mathematical Impacts in Science And Technology, (MIST -17), IJRASET, (November 2017), 109-117.

[12] R. Muthuraj and A. Sasireka, Some Types of Nodal and Edge Regular Anti Fuzzy Graph, International Journal of Fuzzy Mathematical Archive, 14(2) (2017), 365-378.

[13] R. Muthuraj and A. Sasireka, Fuzzy dominator coloring and fuzzy chromatic number on Cartesian product of simple fuzzy graph, Advances in theoretical and applied Mathematics, 11(3) (2016), 245-260.

[14] R. Muthuraj and A. Sasireka, Domination on Anti Fuzzy Graph, International Journal of Mathematical Archive, 9(5) (2018), 82-92.

[15] R. Muthuraj and A. Sasireka, Connected Domination on Anti Fuzzy Graph, Journal of Applied Science and Computations, 5(8) (2018), 18-28.

[16] R. Muthuraj and A. Sasireka, Total Domination on Anti Fuzzy Graph, New Trends in Mathematical Sciences, 6(4) (2018), 28-39.

[17] A. Prasanna, M. A. Rifayathali and S. Ismail Mohideen, Anti-fuzzy graph coloring, international journal of science and advance Research in Technology, 4(4) (2018), 2598-2603.

[18] T. Ramachandran, A. Naseer Ahmed, Dominator Coloring of Middle Graph, IOSR Journal of Mathematics (IOSR-JM), 10(6) (IV) (2014), 38-40.

[19] M.A. Rifayathali, A. Prasanna and S.Ismail Mohideen, Chromatic Excellence in Anti-fuzzy graphs, journal of applied science and computations, 5(7) (july/2018), 305316.

[20] R. Seethalakshmi, and R.B. Gnanajothi, Operations on Antifuzzy graphs, Mathematical Sciences International Research Journal, 5(2) (2016), 210-214.

\author{
$\mathrm{ISSN}(\mathrm{P}): 2319-3786$ \\ Malaya Journal of Matematik \\ ISSN(O):2321-5666

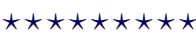

